(C) 2011-18, publisher and licensee JDDT, This is an Open Access article which permits unrestricted
non-commercial use, provided the original work is properly cited

Open $\odot$ Access

Mini Review

\title{
The Effectiveness of Combined Drug Delivery Systems
}

\author{
Zhengwei Zhang \\ School of Science, China Pharmaceutical University, Nanjing, Jiangsu, China, 210009
}

\begin{abstract}
Treatment of tumors with nanoparticles and combined drugs tend to turn effective as it can sustain for a longer time in the recipient's body and also improve the impact. The conjugates that have proven to have high cytotoxicity are suggested in this report. Pegylation is an advanced drug delivery system that maximizes the immunity of the patient and helps in the correct targeting of the affected cells.
\end{abstract}

Keywords: Tumors, Nanoparticles, Drug targeting

Article Info: Received 25 Dec 2018; Review Completed 25 Jan 2019; Accepted 28 Jan 2019; Available online 15 Feb 2019

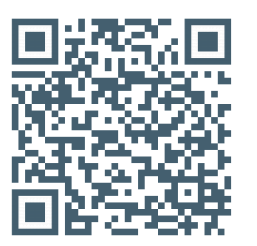

Cite this article as:

Zhang Z, The Effectiveness of Combined Drug Delivery Systems , Journal of Drug Delivery and Therapeutics. 2019; 9(1-

s):377-380 http://dx.doi.org/10.22270/jddt.v9i1-s.2266

*Address for Correspondence:

Zhengwei Zhang, School of Science, China Pharmaceutical University, Nanjing, Jiangsu, China, 210009

\section{Introduction}

Drug delivery system is effective since long to treat patients with different disorders. The drug delivery methods have changed with the introduction of new technologies like nanotechnology ${ }^{1-3}$. Single and combined drug methodologies are used to overcome multidrug resistance and make the nanomedicine infusion process effective. This co-delivery is observed to offer the clinical advantage and improved efficiency thereby adding ease to the treatment of cancer patients ${ }^{4-8}$. This review paper begins with the conjugates that are used for single and combined drug delivery followed by the effectiveness of co-delivery therapy technique. The last section of the report discusses the applications of the codelivery method in the field of biomedicine.

\section{Conjugates for single and combined drug delivery}

The modern society has reported a high number of cases affected by cancer. Chemotherapy is a commonly used treatment for cancer. However, there are issues with the dosage and targeting. Camptothecin is one conjugate that can be used with the inhibitor and diluted with dimethyl sulfoxide. To build a self-assembly structure, camptothecin is conjugated with short oligo ethylene glycol. This can enable the patient to overcome drug resistance ${ }^{9-13}$.
Similar to these conjugates, the nanoparticles that originate from the polymer self-assembly are highly effective. The end conjugate develops a spherical nanoparticle that has up to 8 active drugs. In addition to camptothecin, disulfide bonding is used to crosslink with the nanoparticles and this act as a nano drug delivery system ${ }^{14-17}$. In some instances, paclitaxel and camptothecin are conjugated to develop a beta sheet forming multiple compounds and peptides. The advantage with this bond is that it self-assembles into nanostructures ${ }^{18-}$ 21. This conjugate can release the cancer cause agents and eliminate cervical cancer cell from the body. This conjugation can also help overcome drug resistance.

Not just restricted to the tumor, co-delivery can be a better option for treating breast cancer. Multiple polymers are combined and infused via the nanosized drug delivery system. Mitoxantrone is one anticancer agent that is already in use in the clinic since the past. Now, this is combined with covalent to improve efficiency22-25. Redox-triggered MTO prodrug performs a controlled drug release and improves the therapeutic effect. This amphiphilic MTO prodrug is a combination of MTO and TPGS where the micelles are selfassembled ${ }^{26-29}$. 


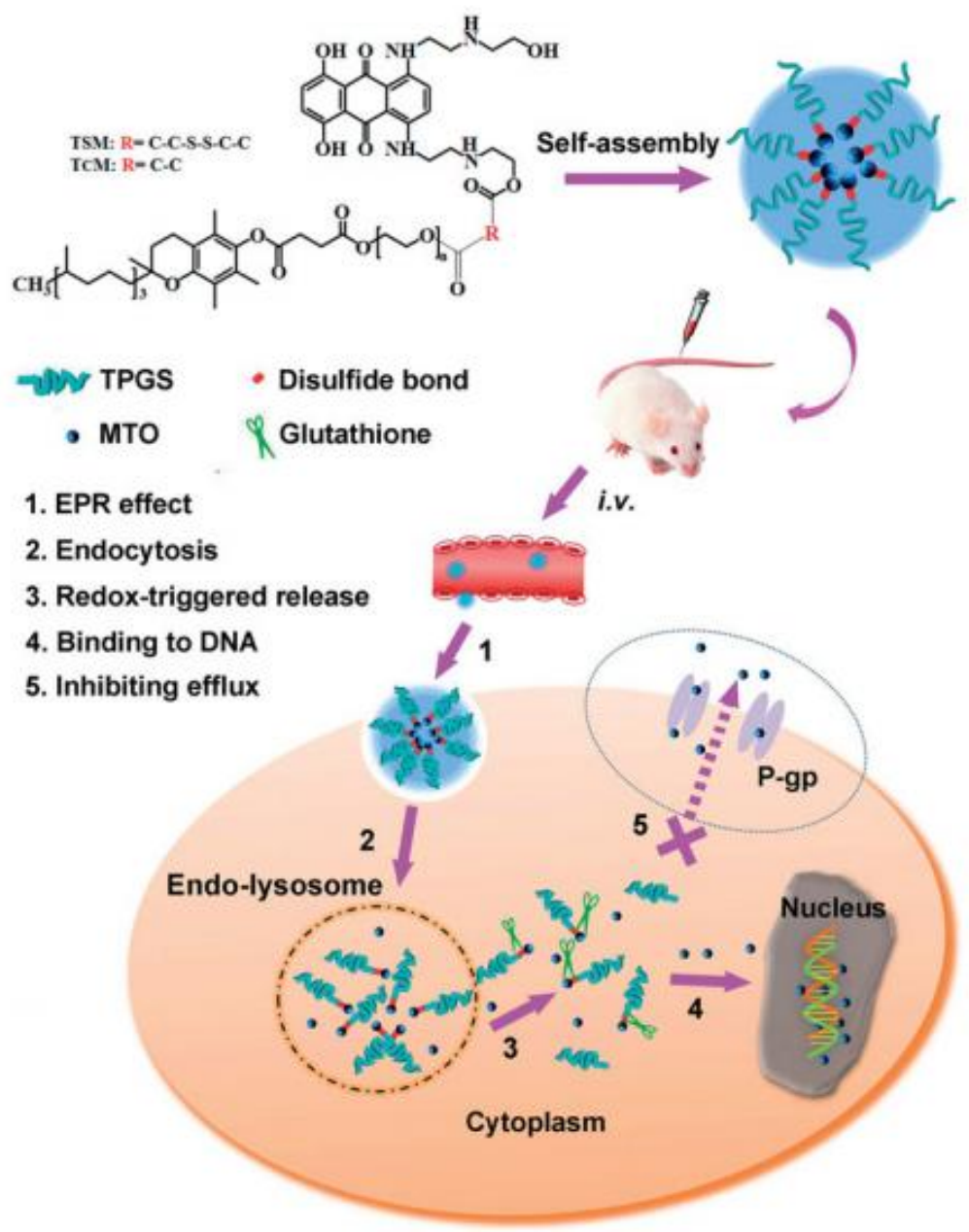

Figure 1: Schematic diagram of self-assembly, tumor targeting, redox-sensitive MTO release responding to the intracellular high level and inhibition of P-gp efflux of TPGS-based prodrug micelles

This combination has brought better apoptosis promoting action. Further, the drug efflux rate is decreased, and drug resistance is controlled for the patient. When a single drug is induced, the patient does not see significant development. However, a co-therapy has multiple advantages with no accumulation of additional cancer or tumor-causing cells ${ }^{30-33}$.

\section{The effectiveness of co-delivery therapy}

Chemotherapy is often hindered due to the multidrug resistance problems faced by cancer patients. The cancer cells cannot tolerate the injuries induced by the drug. Therefore, there is a need for co-administration of various chemotherapeutic agents to be applied in the clinic ${ }^{4}$. It is observed that the co-delivery therapy for cancer is a promising mechanism as the undesirable toxicity is eliminated. Administration of the two drugs - fluorouracil and paclitaxel can improve efficiency in the treatment of MDR cancer ${ }^{34-38 .}$.
There are multiple ways to enable co-delivery - inducing dual chemotherapeutic drugs, with MDR inhibitor and with a sensitizer. The MDR inhibitor is a combination of cytotoxic drugs and many efflux pump inhibitors. This combination can maintain cytotoxicity and improve pharmacokinetic properties. The most promising inhibitor is tariquidar as this can sensitize those resistant cell lines and control the overexpression of P-gp $22,24,39,40$.

On the other hand, sensitizer compounds can fix the dysfunctional apoptotic signaling with chemotherapeutic drugs. Curcumin is yet another polyphenol that engages in anticancer activity and also down regulates the pathways from functioning and progressing the cancer cells further to other parts. It is observed that the co-delivery has a higher potential to control MDR and this only requires the proper selection of the nanotherapeutic delivery system to choose inhibitor/ sensitizer/ modulator ${ }^{9-11,37 . ~}$ 
Table 1. Summary of Listed Nanoparticles with Dual Drug Delivery to Overcome Multidrug Resistance.

\begin{tabular}{|c|c|c|c|c|}
\hline Materials & Agent 1 & Agent 2 & Cancer cell lines & Ref \\
\hline $\begin{array}{l}\text { Poly(z-caprolactone) and poly(ethyl ethylene } \\
\text { phosphate) }\end{array}$ & Doxorubicin & NA & MCF-7/ADR & (38) \\
\hline $\begin{array}{l}\text { Poly(lactic-co-glycolic acid) and polyethylene } \\
\text { glycol }\end{array}$ & Vincristine & NA & MCF-7/ADR & $(42)$ \\
\hline Pluronic-P105 & Doxorubicin & Paclitaxel & MCF-7/ADR & (46) \\
\hline $\begin{array}{l}\text { Poly(ethylene oxide)-poly(propylene oxide)- } \\
\text { poly( } \varepsilon \text {-caprolactone) }\end{array}$ & Docetaxel & Chloroquine & MCF-7 and MCF-7/ADR & (47) \\
\hline VE and tocopherol poly(ethylene glycol)succinate & Paclitaxel & 5-Fluorouracil & KB-8-5 and KB-3-1 & (48) \\
\hline Poly (lactic-co-glycolic acid) & Docetaxel & TPGS & HeLa & (50) \\
\hline EPC, DOTAP, cholesterol and PEG2KPE & Paclitaxel & Tariquidar & SKOV-3 and SKOV-3TR & (51) \\
\hline CEA and AHM & Doxorubicin & Verapamil & $\mathrm{NCl} / \mathrm{ADR}-\mathrm{RES}$ & (53) \\
\hline Chitosan & Doxorubicin & Pyrrolidinedithiocarbamate & HepG-2 & (54) \\
\hline TPGS2000 and PEG2000-DSPE & Doxorubicin & Curcumin & MCF7/ADR & (55) \\
\hline Poly(D,L-lactide-co-glycolide) & Doxorubicin & Curcumin & K562 & (56) \\
\hline $\begin{array}{l}\text { 1-Palmitoyl-2-azelaoyl-sn-glycero-3- } \\
\text { phosphocholine }\end{array}$ & Doxorubicin & Ceramide & P388/ADR & (57) \\
\hline $\begin{array}{c}\text { Precirol ATO 5,Squalene, SPC, Tween-80 and } \\
\text { DOTAP }\end{array}$ & Doxorubicin/Paclitaxel & $\begin{array}{l}\text { siRNA targeting MRP1 and } \\
\mathrm{BCL} .2\end{array}$ & A549 & (62) \\
\hline PAMAM and PEG-2K-DOPE & Doxorubicin & siRNA targeting GFP & A549 cells and C166-GFP & (63) \\
\hline
\end{tabular}

\section{Pegylated drug delivery applications}

Drug delivery has now got different methods to reach the targeted cells and use inhibitors to control the activity of attacking molecule. Pegylation is a procedure where polyethylene glycol (PEG) (hydrophilic) is attached to the therapeutic molecule. This attachment improves hydrophilicity and also keeps up the activity of enzymes 29,30 . Pegylation typically includes hydroxyl groups, and PEG is attached to liposome/protein/peptide/polymersome. The application of PEG does not show high toxicity level. It has so far proved to be safe to the humans.

The only drawback with this delivery system is the choice of molecules to influence the pharmacokinetics. Pegylation is a favorite technique to deliver drugs to the end body ${ }^{30}$. It is a model that is used in genetic engineering as well as several in vivo and in vitro applications. The other advantage with pegylation is that it can increase the half-life of vectors. As a result, the circulation times increases and the impact is longer. The concerned health problem is reduced.

\section{Conclusion}

The types of drug deliveries are numerous in the market. Nanomedicine is a field that encourages a play with nanoparticles to treat multiple health disorders. From the observation in this review sheet, it is known that co-delivery of therapeutic drugs can increase the effectiveness and the targeted delivery is feasible. The conjugate has to be chosen carefully. This drug delivery requires expert knowledge. Otherwise, it has a widespread application in the treatment of cancer and tumors and can be the future of the medical field.

\section{References}

1. Kang, C., Qin, J., Osei, W. \& Hu, K. Age-dependent Mitochondrial Targeting Of Protein Kinase C Epsilon In Cardioprotection. The FASEB Journal 2017.

2. Han, R., Sun, Y., Kang, C., Sun, H. \& Wei, W. Amphiphilic dendritic nanomicelle-mediated co-delivery of 5-fluorouracil and

ISSN: 2250-1177 doxorubicin for enhanced therapeutic efficacy. Journal of Drug Targeting 2017; 25:140-148.

3. Duan, Y., et al. Bioactivity evaluation-based ultra highperformance liquid chromatography coupled with electrospray ionization tandem quadrupole-time-of-flight mass spectrometry and novel distinction of multi-subchemome compatibility recognition strategy with Astragali Radix-Fructus Corni herbpair as a case study. J Pharm Biomed Anal 2016; 129:514-534.

4. Sun, Y., et al. Co-delivery of dual-drugs with nanoparticle to overcome multidrug resistance. European Journal of BioMedical Research 2016; 2:12-18.

5. Ai, R., et al. Comprehensive epigenetic landscape of rheumatoid arthritis fibroblast-like synoviocytes. Nat Commun 2018; 9:1921.

6. Fan, S., et al. Computationally expanding infinium HumanMethylation450 BeadChip array data to reveal distinct DNA methylation patterns of rheumatoid arthritis. Bioinformatics 2016; 32:1773-1778.

7. Liu, F., Sun, Y. \& Kang, C. Controlling Amphiphilic Functional Block Copolymers' Self-Assembly: From Structure to Size. (2016).

8. Song, L., et al. Crocetin inhibits lipopolysaccharide-induced inflammatory response in human umbilical vein endothelial cells. Cellular Physiology and Biochemistry 2016; 40:443-452.

9. Sun, Y., Kang, C., Liu, F. \& Song, L. Delivery of antipsychotics with nanoparticles. Drug Development Research 2016; 77:393-399.

10. Kang, C., et al. Delivery of nanoparticles for treatment of brain tumor. Current Drug Metabolism 2016; 17:745-754.

11. Xue, X., et al. Discovery of novel inhibitors disrupting HIF$1 \alpha /$ von Hippel-Lindau interaction through shape-based screening and cascade docking. PeerJ 2016; 4:e2757.

12. Hersch, S.J., et al. Divergent protein motifs direct elongation factor P-mediated translational regulation in Salmonella enterica and Escherichia coli. MBio 2013; 4:e00180-00113.

13. Shuhong, X., et al. Dynamic expression of AQP4 in early stageof ischemia/reperfusion rats and cerebral edema. Chinese Pharmacological Bulletin 2016; 32:1433-1441. 
14. Peng, J., et al. Enhanced Liver Regeneration After Partial Hepatectomy in Sterol Regulatory Element-Binding Protein (SREBP)-1c-Null Mice is Associated with Increased Hepatocellular Cholesterol Availability. Cellular Physiology and Biochemistry 2018; 47:784-799.

15. Yang, Z., et al. Functional exosome-mimic for delivery of siRNA to cancer: in vitro and in vivo evaluation. Journal of Controlled Release 2016; 243:160-171.

16. Kang, C., Hernandez, V.A. \& Hu, K. Functional interaction of the two-pore domain potassium channel TASK-1 and caveolin-3. Biochimica et Biophysica Acta (BBA)-Molecular Cell Research 2017; 1864:1537-1544).

17. Waller, A.P., et al. GLUT12 functions as a basal and insulinindependent glucose transporter in the heart. Biochimica et Biophysica Acta (BBA)-Molecular Basis of Disease 2013; 1832:121-127.

18. Li, Q., et al. Identification by shape-based virtual screening and evaluation of new tyrosinase inhibitors. PeerJ 2018; 6:e4206.

19. Chen, Y., et al. Identification of 4-aminoquinoline core for the design of new cholinesterase inhibitors. PeerJ 2016; 4:e2140.

20. Kang, C. \& Hu, K. Impact of hypoxia in the expression and regulation of the TASK-1 potassium channel in cardiac myocytes. The FASEB Journal 2016; 30:lb598-lb598.

21. Kang, C. Ion channels, protein kinase $C$ and caveolae in cardioprotection, (The Ohio State University, 2015).

22. Qiao, H., et al. Redox-triggered mitoxantrone prodrug micelles for overcoming multidrug-resistant breast cancer. Journal of drug targeting 2018; 26:75-85.

23. Kang, C., Qin, J., Osei, W. \& Hu, K. Regulation of protein kinase Cepsilon and its age-dependence. Biochemical and Biophysical Research Communications 2017; 482:1201-1206.

24. Sun, Y., et al. RGD Peptide-Based Target Drug Delivery of Doxorubicin Nanomedicine. Drug development research 2017; 78:283-291.

25. Kang, C. \& Hu, K. Role of caveolin-3 in adenosine-induced increase in mitochondrial PKCE. The FASEB Journal 2013; 27:1191.1197-1191.1197.

26. Kang, C. \& Hu, K. Modulation of the two-pore domain potassium channel TASK-1 by caveolin-3. The FASEB Journal 2015; 29:845.814.

27. Davis, M.E., Chen, Z.G. \& Shin, D.M. Nanoparticle therapeutics: an emerging treatment modality for cancer. Nat Rev Drug Discov 2008; 7:771-782.
28. Kang, C., Sun, Y., Wang, M. \& Cheng, X. Nanosized camptothecin conjugates for single and combined drug delivery. European Journal of BioMedical Research 2016; 2:8-14.

29. Qiao, H., et al. Orally delivered polycurcumin responsive to bacterial reduction for targeted therapy of inflammatory bowel disease. Drug Delivery 2017; 24:233-242.

30. Liu, F., Sun, Y., Kang, C. \& Zhu, H. Pegylated Drug Delivery Systems: From Design to Biomedical Applications. Nano LIFE 2016; 6:1642002.

31. Sun, Y., Kang, C., Yao, Z., Liu, F. \& Zhou, Y. Peptide-Based Ligand for Active Delivery of Liposomal Doxorubicin. Nano Life 2016; $6: 1642004$.

32. Yeh, C.Y., Hsiao, J.K., Wang, Y.P., Lan, C.H. \& Wu, H.C. Peptideconjugated nanoparticles for targeted imaging and therapy of prostate cancer. Biomaterials 2016; 99:1-15.

33. Fan, S., Huang, K., Ai, R., Wang, M. \& Wang, W. Predicting CpG methylation levels by integrating Infinium HumanMethylation450 BeadChip array data. Genomics 2016; 107:132-137.

34. Cheng, X. \& Lee, R.J. The role of helper lipids in lipid nanoparticles (LNPs) designed for oligonucleotide delivery. Adv Drug Deliv Rev 2016; 99:129-137.

35. Sun, Y. \& Kang, C. Self-Assembly of Peptides into Hydrogel. Journal of Organic \& Inorganic Chemistry 2016; 2:5.

36. Yao, Z., Sun, Y. \& Kang, C. Structure and self-assembly of multicolored Naphthalene Diimides Semiconductor. Nano LIFE 2016; 6: 1642007.

37. Cheng, X., et al. T7 Peptide-Conjugated Lipid Nanoparticles for Dual Modulation of Bcl-2 and Akt-1 in Lung and Cervical Carcinomas. Molecular pharmaceutics 2018; 15:4722-4732.

38. Zhong, X., Sun, Y., Kang, C. \& Wan, G. The theory of dielectrophoresis and its applications on medical and materials research. European Journal of BioMedical Research 2017; 2:7-11.

39. Yung, B.C., et al. Lipid nanoparticles composed of quaternary amine-tertiary amine cationic lipid combination (QTsome) for therapeutic delivery of AntimiR-21 for lung cancer. Molecular pharmaceutics 2016; 13:653-662.

40. Cheng, X., et al. Lipid Nanoparticles Loaded with an Antisense Oligonucleotide Gapmer Against Bcl-2 for Treatment of Lung Cancer. Pharmaceutical research 2017; 34:310-320. 